\title{
The Workshop on Animal Botulism in Europe
}

Skarin, Hanna; Tevell Åberg, Annica; Woudstra, Cédric; Hansen, Trine; Löfström, Charlotta; Koene, Miriam; Bano, Luca; Hedeland, Mikael; Anniballi, Fabrizio; De Medici, Dario

Total number of authors:

11

Published in:

Biosecurity and Bioterrorism

Link to article, DOI:

$10.1089 / \mathrm{bsp} .2012 .0076$

Publication date:

2013

Document Version

Publisher's PDF, also known as Version of record

Link back to DTU Orbit

Citation (APA):

Skarin, H., Tevell Åberg, A., Woudstra, C., Hansen, T., Löfström, C., Koene, M., Bano, L., Hedeland, M., Anniballi, F., De Medici, D., \& Olsson Engvall, E. (2013). The Workshop on Animal Botulism in Europe. Biosecurity and Bioterrorism, 11(1), S183-S190. https://doi.org/10.1089/bsp.2012.0076

\section{General rights}

Copyright and moral rights for the publications made accessible in the public portal are retained by the authors and/or other copyright owners and it is a condition of accessing publications that users recognise and abide by the legal requirements associated with these rights.

- Users may download and print one copy of any publication from the public portal for the purpose of private study or research.

- You may not further distribute the material or use it for any profit-making activity or commercial gain

- You may freely distribute the URL identifying the publication in the public portal 


\section{The Workshop on Animal Botulism in Europe}

Hanna Skarin, Annica Tevell Åberg, Cédric Woudstra, Trine Hansen, Charlotta Löfström, Miriam Koene, Luca Bano, Mikael Hedeland, Fabrizio Anniballi, Dario De Medici, and Eva Olsson Engvall

A workshop on animal botulism was held in Uppsala, Sweden, in June 2012. Its purpose was to explore the current status of the disease in Europe by gathering the European experts in animal botulism and to raise awareness of the disease among veterinarians and others involved in biopreparedness. Animal botulism is underreported and underdiagnosed, but an increasing number of reports, as well as the information gathered from this workshop, show that it is an emerging problem in Europe. The workshop was divided into 4 sessions: animal botulism in Europe, the bacteria behind the disease, detection and diagnostics, and European collaboration and surveillance. An electronic survey was conducted before the workshop to identify the 3 most needed discussion points, which were: prevention, preparedness and outbreak response; detection and diagnostics; and European collaboration and surveillance. The main conclusions drawn from these discussions were that there is an urgent need to replace the mouse bioassay for botulinum toxin detection with an in vitro test and that there is a need for a European network to function as a reference laboratory, which could also organize a European supply of botulinum antitoxin and vaccines. The foundation of such a network was discussed, and the proposals are presented here along with the outcome of discussions and a summary of the workshop itself.

$\mathrm{O}$ N JUNe 7 and 8, 2012, an animal botulism workshop was held in Uppsala, Sweden. The workshop was hosted by the Swedish National Veterinary Institute and organized through the European Union (EU)-funded project AniBioThreat.

\section{BACKGROUND}

Botulism is a paralytic disease caused by botulinum neurotoxins, which are produced mainly by the bacterial species Clostridium botulinum. These neurotoxins affect

Hanna Skarin, MSc, is a Research Assistant, Department of Bacteriology, National Veterinary Institute (SVA), Uppsala, Sweden, and Department of Biomedical Sciences and Veterinary Public Health, Swedish University of Agricultural Sciences (SLU), Uppsala, Sweden. Annica Tevell Åberg, PhD, is a Senior Researcher, and Mikael Hedeland, PhD, is Associate Professor and Deputy Head of the Department, Department of Chemistry, Environment and Feed Hygiene, National Veterinary Institute (SVA), Uppsala, Sweden. Cédric Woudstra, MSc, is an Engineer, French Agency for Food, Environmental and Occupational Health Safety (ANSES), Food Safety Laboratory, Maisons-Alfort, France. Trine Hansen, MSc, is a PhD student, and Charlotta Löfström, PhD, is an Assistant Professor, both at the National Food Institute, Technical University of Denmark (DTU), Søborg, Denmark. Miriam Koene, DVM, is a Scientist, Central Veterinary Institute of Wageningen University and Research Centre (CVI), Lelystad, the Netherlands. Luca Bano, $\mathrm{PhD}$, is Veterinary Officer, Istituto Zooprofilattico Sperimentale delle Venezie (IZSVe), Veterinary Diagnostic Laboratory of Treviso, Treviso, Italy. Fabrizio Anniballi, CLT, is a Laboratory Technician, and Dario De Medici, PhD, is Senior Researcher, both at Istituto Superiore di Sanità (ISS), Department of Veterinary Public Health and Food Safety, Rome, Italy. Eva Olsson Engvall, PhD, is Associate Professor, Department of Bacteriology, National Veterinary Institute (SVA), Uppsala, Sweden, and Department of Biomedical Sciences and Veterinary Public Health, Swedish University of Agricultural Sciences (SLU), Uppsala, Sweden. 
animals as well as humans worldwide and are included in the Centers for Disease Control and Prevention (CDC) list of potential biothreat agents. ${ }^{1} C$. botulinum is a strictly anaerobic spore-forming bacterium found in soil around the world. ${ }^{2}$ It produces neurotoxin in environments that permit germination of the spores, such as canned food and silage. ${ }^{3,4}$ The accessibility and dissemination potential of C. botulinum spores in combination with the high health impact on both humans and animals also make the bacterium a biological risk agent. ${ }^{1}$ Botulism is usually caused by ingestion of preformed toxin, but there are also cases caused by toxicoinfection. The toxicoinfectious form, which is mainly connected with wound botulism and botulism in young animals, infants, and birds, is caused by botulinum neurotoxins produced in situ after germination of spores in wounds or the intestine.

Strains belonging to the species $C$. botulinum can be divided into 4 physiological groups, I through IV.' Groups I and II cause both human and animal botulism, whereas group III is mainly connected with animal botulism. Although considered a zoonotic disease, human botulism attracts more attention than animal botulism, which has resulted in stricter national and international regulations and control measures against human botulism compared to animal botulism. For example, thanks to international regulations and networks, there are surveillance data for human botulism cases, whereas the status of animal botulism is vague. Considering the economic impact and the large number of animals that die during an animal botulism outbreak, it is important to focus also on animal botulism. And in terms of biopreparedness, it is essential to consider both human and animal health.

\section{Purpose and Outline of the Workshop}

The purpose of the workshop was to explore the current status of animal botulism in Europe and to discuss important issues regarding laboratory work and diagnostics, control measures, and biopreparedness by gathering the European experts in the area of animal botulism. Another important aspect of this workshop was to take the opportunity to share the information from AniBioThreat on animal botulism with European veterinarians and scientists working on animal botulism outside the project. The participants were veterinarians and scientists from 11 European countries: Belgium, Denmark, Finland, Germany, Great Britain, France, Italy, Norway, Spain, Sweden, and the Netherlands; 1 invited keynote speaker was from Japan. Abstracts describing the situation of animal botulism in the past decade were collected from participating countries as a complement to the workshop. ${ }^{6}$ An electronic survey (QuestBack) was conducted before the workshop to collect information about key issues and to identify relevant discussion points.

The workshop was divided into 4 sessions. The first session, Animal Botulism in Europe, aimed to explore the current status of the disease in Europe and to discuss prac- tical measures taken in European countries during botulism outbreaks. Sessions 2 and 3, The Bacteria Behind the Disease and Detection and Diagnostics, aimed to inform the participants about genomic characterizations, epidemiology, and detection method developments that were performed in the AniBioThreat project. A further objective was to implement those methods at other laboratories in Europe by providing demonstrations of methods. These 2 sessions also included discussions about laboratory work and future diagnostic needs. Session 4, European Collaboration and Surveillance, was intended to highlight EU projects that focus on botulism as examples of European collaboration and to discuss implementation of control measures and surveillance of animal botulism at a European level.

This meeting report provides synopses of the sessions, including key information given at presentations and collected from the abstract book and survey, and the outcomes of discussions in the form conclusions and proposals for future work on animal botulism in Europe. The presenters are listed at the end of this article.

\section{Opening OF THE WORKSHOP}

The workshop was opened by Hanna Skarin and Jeffrey Skiby, followed by a presentation about the AniBioThreat project by coordinator Rickard Knutsson. Dr. Knutsson presented the project background and introduced the core issue of the project, which is to bridge the fields of research, security, and safety in order to improve the EU's capacity to counter biological animal biothreats. Viveca Båverud then introduced the topic of animal botulism from a veterinary perspective. She described the classical signs of flaccid paralysis caused by the botulinum neurotoxin and the variety of symptoms in different animal species. Keynote speaker Shunji Kozaki from Osaka Prefecture University in Japan was invited to present research regarding the organization and biological properties of the botulinum neurotoxins produced by $C$. botulinum group III.

Synopsis of Animal Botulism in Europe SESSION

\section{Current Status of the Disease in Europe}

Presentations in this session covered the current status of the disease in Norway (Simon Hardy), Spain (Ibone Anza Gomez), Italy (Luca Bano), France (Rozenn Souillard and Caroline Le Maréchal), Germany (Helge Böhnel, Frank Gessler, and Birgit Schwagerick), and Sweden (Hanna Skarin and Gunilla Blomqvist). The focus in all presentations, as well as in the abstracts, which described the status also in Belgium, Denmark, the Netherlands, and the United Kingdom, was on botulism in cattle or avian 
species. Botulism in dogs, horses, minks, and small ruminants was also mentioned. So-called visceral or chronic botulism was reported from Germany and Denmark. This condition affecting cattle is considered to be caused by a proliferation of C. botulinum group III strains in the gut (toxicoinfection). Although the use of botulinum vaccines was reported to have beneficial effect in some herds with visceral botulism, the existence of this specific clinical form of botulism is still controversial. ${ }^{6}$

Difficulties in evaluating the disease situation and gathering epidemiologic data were discussed. Several reasons were offered, including the limited knowledge of the disease among veterinary practitioners, the lack of laboratories authorized to perform the reference method for botulism diagnostics (the mouse bioassay), and farmers' concerns about diagnosing the disease because of the trade restrictions that result if botulism is confirmed. It was concluded that one of the most important factors leading to an underestimation of the disease is that the diagnosis of botulism often is made by veterinary practitioners and based solely on clinical signs without laboratory confirmation (Figure 1). Furthermore, the lack of therapeutic (antitoxins) or prophylactic (vaccines) tools needed to combat animal botulism outbreaks in many European countries discourages requests for laboratory confirmation. As a consequence, many cases are not reported, since the laboratory usually reports confirmed botulism cases to national authorities. Moreover, animal botulism is not a notifiable disease in most European countries (unlike human botulism), which also contributes to cases never being reported.
In spite of the fact that animal botulism is an underdiagnosed and underreported disease, it is an emerging problem in several European countries. An increase of animal botulism cases during the past decade was reported at the workshop, in the abstracts, ${ }^{6}$ and in other reports. ${ }^{7-10}$

\section{Prevention, Preparedness, and Outbreak Response}

To summarize key information from the presentations in this session regarding prevention, preparedness, and outbreak response:

- Spores of C. botulinum are not frequently or constantly present in Scandinavian poultry holdings, even at times when outbreaks have occurred. Therefore, it is likely that an outbreak is preceded by a recent introduction of spores to the flock.

- Identified sources of C. botulinum spores for poultry and cattle are feed, rice hulls used as a litter component, carcasses, rodents, soil, and hay packaged in plastic.

- Identified risk factors for botulism in poultry are a low biosafety barrier, no acidification or chlorination of drinking water, and no pest control.

- Quick removal of carcasses during outbreaks is effective in slowing down the rate of the outbreak, both in wild and domestic avian species.

- Administration of ampicillin in drinking water is an effective method of decreasing the mortality rate in poultry outbreaks. However, clinical signs of botulism may

\section{In your country, are laboratory analyses performed to diagnose animal botulism?}

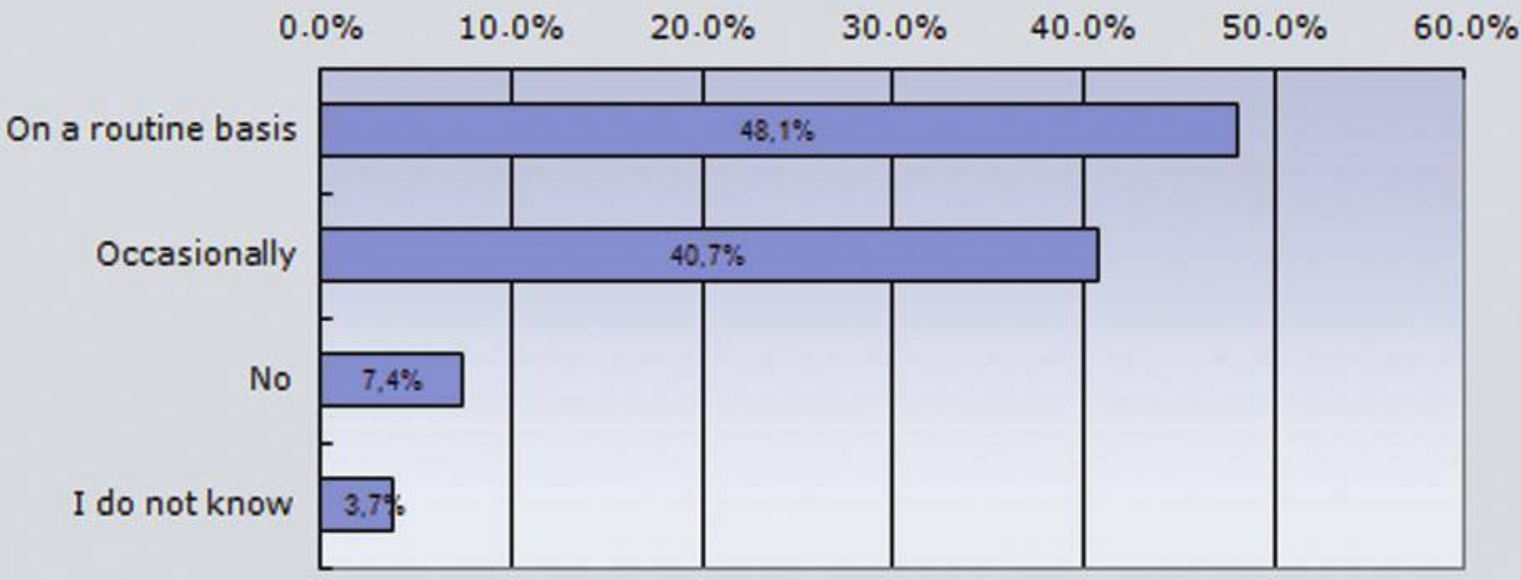

Figure 1. Laboratory Confirmation of Animal Botulism. The figures show the results from question 2 in the survey. The results are shown as a percentage of the 27 answers from survey participants, who are from Belgium, Denmark, Finland, Germany, Great Britain, France, Italy, Norway, Spain, Sweden, and the Netherlands. Fewer than $50 \%$ of the participants in the survey responded that laboratory analyses are performed on a routine basis to confirm botulism. This result indicates that there are many cases of botulism in animals that will never be reported. Color images available online at www.liebertpub.com/bsp 
reappear before slaughter when animals are not allowed to be treated with antibiotics.

The matter of asymptomatic infection was also discussed. It is known that poultry can carry spores and toxin without showing any symptoms. ${ }^{11,12}$ Such carriers pose a risk to other animals, especially if poultry carcasses or manure are used for fertilizing pastures or grass that will be used for silage. If recommended practices were followed, the spread of spores from chickens could be prevented. ${ }^{13}$ What is less well known is how frequently other farm and wild animals act as carriers of spores of $C$. botulinum. This should be further explored as it could be another risk factor for botulism outbreaks.

It was concluded that there is a need for guidelines and control measures for handling a botulism outbreak-for example, regarding incineration of dead animals, disinfection of farms, and dealing with manure from the farm. There is also a need for better animal feed quality management.

Toxoid botulinum vaccines are commercially available for use in mink ( $C$. botulinum type $C$ toxin), cattle and sheep (both $C$. botulinum type $\mathrm{C}$ and type $\mathrm{D}$ toxin), and horses (C. botulinum type B toxin). However, those vaccines, except for some produced and used at the national level, are imported from the US, South Africa, and Australia. The results from the survey showed that mink is the only animal species routinely vaccinated against botulinum toxin in Europe. Cattle are sometimes vaccinated in outbreak situations, and horses occasionally are vaccinated during outbreaks or if they are kept in pastures in areas with a history of botulism. In Italy, pheasants are sometimes vaccinated with vaccine intended for mink. Vaccination of cattle was considered to be effective in outbreak situations if administrated quickly. Unfortunately, vaccine stocks for cattle are not available in all European countries, and it may be time-consuming to receive import permits for vaccines. The availability of antiserum and vaccines in Europe was discussed, and it was concluded that there is a need for a European antitoxin bank with sera against the different toxin types, as well as a centralized European commercial vaccine production facility. The fact that most of the botulinum vaccines used in Europe are imported makes European livestock vulnerable in a crisis situation, whether there is a biothreat against animals or an increase in outbreaks due to natural causes.

\section{Synopsis of the Session C. botulinum: The Bacteria Behind the Disease}

Keynote speaker Ian Poxton from the University of Edinburgh in Scotland introduced the complex question of whether $C$. botulinum group III and $C$. novyi should belong to the same species. He also talked about equine grass sickness, which causes malfunctions of the autonomic nervous system of horses. Little is known about the etiology of the disease, but it is thought to be caused by $C$. botulinum group III or C. novyi. ${ }^{14}$ The possibility that it could be due to a toxicoinfection in a way similar to that proposed to be the cause of visceral botulism was discussed. It was also hypothesized that these types of diseases may be caused by toxicoinfection of botulinum strains that have lost the ability to produce botulinum neurotoxin.

In their presentation, Bo Segerman and Hanna Skarin talked about the genetic diversity within the pan-genome of C. novyi and group III of C. botulinum. They concluded that the pathogenicity of strains is more dependent on the plasmids they contain than to which genotypic group they belong. C. botulinum group III harbors many plasmids and prophages containing several toxin genes in addition to the botulinum neurotoxin, ${ }^{15}$ which supports the hypothesis that a strain that has lost the ability to produce botulinum neurotoxin can still be pathogenic.

\section{Laboratory Work on C. botulinum Group III}

The issue of strains losing the bacteriophage during preenrichment was discussed, since this leads to a negative result by current diagnostic detection methods, which are based on confirmation of the neurotoxin or the neurotoxin gene. It was experienced that some strains lose the bacteriophage more easily than others, but this must be further explored. A chromosomal marker can be included in the diagnostic PCR assays in order to detect the bacterium, but it is not always useful to detect a toxin-negative strain since it does not provide supportive data for confirmation of the disease.

Another issue that was discussed at the meeting was that of strain isolation from clinical or environmental samples. Some laboratories successfully isolate strains by direct cultivation on blood or egg agar plates and by recognition of the colony morphology. One laboratory used an immunomagnetic separation technique and considered that to be helpful. However, due to several factors such as poor growth on agar plates and being a strict anaerobe, it was concluded that $C$. botulinum is a difficult organism to isolate.

It was concluded from the survey that most participating institutes perform laboratory work on C. botulinum group III strains in BSL-2 laboratories, although a few work in BSL-3 laboratories.

Synopsis of the Detection and Diagnostics Session

\section{Toxin-based Detection and Diagnostic Developments}

Keynote speaker Miia Lindström from the University of Helsinki in Finland gave an introduction to botulism diagnostics, including the different steps in the mouse bioassay, which is used to diagnose botulism, and pointed out the drawbacks of the method.

Biosecurity and Bioterrorism: Biodefense Strategy, Practice, and Science 
Two different alternative methods for botulinum toxin detection were presented by Jan Langeveld and Annica Tevell Åberg. Both alternative methods demonstrate toxin activity through cleavage of synthetic peptides, which mimic the proteins involved in the SNARE complex where the toxins act in vivo. Jan Langeveld presented an assay that combines botulinum neurotoxin capture on synaptosomes and immunochemical analysis of the peptide cleavage products. ${ }^{16}$ Annica Tevell Åberg presented an endopeptidase method that uses an antibody capture of the botulinum neurotoxin followed by determination of its activity and differentiation between different serotypes by mass spectrometric detection of the cleaved peptides. The latter assay, called Endopep-MS, was developed at CDC. ${ }^{17-19}$ Both methods are currently being optimized and validated with the goal of replacing the mouse bioassay.

The mouse bioassay is still considered to be the gold standard method for laboratories to confirm botulism and is thus by far still the most common method used by European laboratories, as reported in the survey conducted in connection with the workshop (Figure 2). The urgency of replacing the mouse bioassay with an in vitro test was raised, both for ethical reasons and because several laboratories have experienced increased difficulties in getting permission to continue performing the mouse bioassay. While developing and validating replacement methods, it is important to decrease the number of mice used, and it was suggested that the mouse bioassay should be used only to confirm active botulinum toxin, but that serotype should be determined by alternative methods. It was agreed that it is important to have a method that detects active toxin and all serotypes of toxins with equal sensitivity. From a clinical viewpoint, fast detection is also important since administration of antitoxin has to be done within the first 24 hours to be effective.

\section{DNA-based Detection}

Joakim Ågren presented the software Gegenees (www. gegenees.com), which can be used for comparative analyses of whole genome sequence data and to define unique genomic signatures for specific target groups. ${ }^{20}$ The program is useful when designing specific detection methods. Cedric Woudstra presented the GeneDisc technology and the 2 prototypes for detection of botulinum neurotoxin gene of type $\mathrm{C}, \mathrm{D}$, and mosaic types $\mathrm{C} / \mathrm{D}$ and $\mathrm{D} / \mathrm{C}$ toxin. ${ }^{21}$ The Gegenees program and GeneDisc technology were also demonstrated for participants to get a hands-on experience from using the different methods. It was agreed that gene detection methods are suitable for detection of $C$. botulinum in research settings and for screening of samples in order to minimize the use of the mouse bioassay.

\section{Synopsis of the European Collaboration and Surveillance Session}

The EU-financed AniBioThreat project is a good example of European collaboration regarding animal botulism, and

\section{If laboratory analyses are performed, which diagnostic method(s) are used to confirm diagnosis of botulism?}

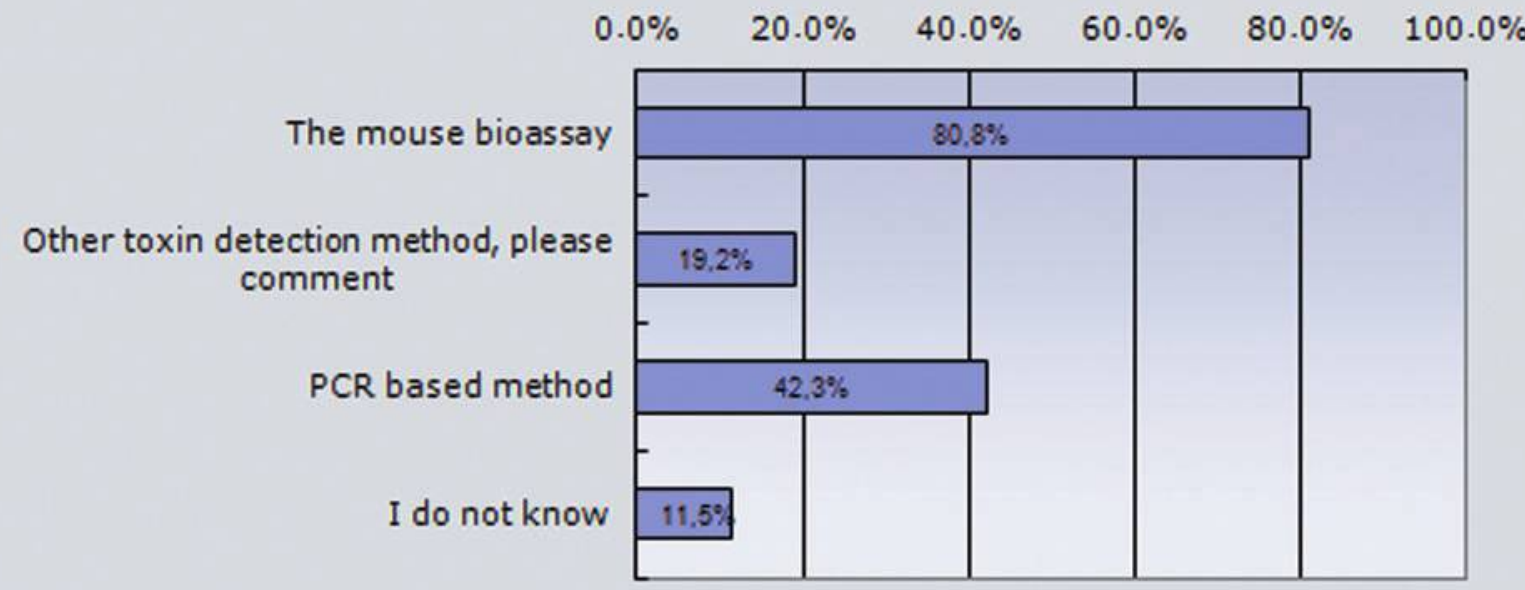

Figure 2. Laboratory Confirmation of Animal Botulism. The figure shows the results from question 3 in the survey. The results are shown as a percentage of the 27 answers from survey participants, who are from Belgium, Denmark, Finland, Germany, Great Britain, France, Italy, Norway, Spain, Sweden, and the Netherlands. The mouse bioassay is still the gold standard method in botulism diagnosis, which is reflected in the answers to question 3. The other toxin detection method that was mentioned in the comments was the enzyme-linked immunosorbent assay (ELISA). PCR-based methods are also used to some extent in European laboratories for confirmation of botulism. Color images available online at www.liebertpub.com/bsp 
the work performed so far was summarized by task leader Dario De Medici. The overall objective was to improve methods for detection of $C$. botulinum in animal, feed, environmental, and food samples. Several detection methods, PCR based and toxin based, have been developed in the project, and methods will be validated and established by performing ring trials in the partner institutes, all in order to increase the EU's capacity to respond to animal bioterrorism threats. Andreas Rummel presented the EU project Establishment of Quality Assurances for the Detection of Biological Toxins of Potential Bioterrorism Risk (EQuaTox), which started in 2012 (http://equatox.net). The project aims to harmonize and standardize detection methods for biological toxins, such as botulinum toxin.

Most participants in the survey believed that there is a need for a European network to function as a reference laboratory for botulism. There is currently no World Organization for Animal Health (OIE) reference laboratory or collaborating center for animal botulism and, to our knowledge, no other reference laboratory at the European or international level focusing on animal botulism. The structure of such a reference lab was further discussed. As botulism is a disease that can affect both humans and animals, it was decided to propose a structure that covers both food and animal safety. Instead of centralizing the duties of a reference laboratory in 1 institute, it was proposed to initiate a reference laboratory network consisting of several institutes. The purpose of such a network should be to standardize and harmonize methods, by, for example, offering reference material, and to provide expertise and diagnostic facilities. Laboratories that cannot perform the required tests should be able to send their samples to the network laboratories for testing.

The need for a surveillance system for animal botulism was also discussed. It was suggested that botulism should be added to the OIE listed diseases (http://www.oie.int/ animal-health-in-the-world/oie-listed-diseases-2012/), which means that it would be regarded as a notifiable disease worldwide. Botulism is included in the B list of zoonoses and zoonotic agents to be monitored according to the epidemiologic situation as stated in the EU Zoonoses Directive, ${ }^{22}$ and $\mathrm{EU}$ member states can therefore decide to establish a surveillance system if required. According to the same directive, foodborne outbreaks in humans must be reported. The European Center of Disease Prevention and Control (ECDC) identifies and communicates threats to human health in terms of infectious diseases by collecting surveillance data from member states and compiling it into annual epidemiologic reports. Regarding botulism, these reports take into account only confirmed human botulism cases, since that is currently the only accessible surveillance data in the EU. When it comes to botulism in animals, it is up to the European Commission together with member states to decide if surveillance will be organized across the EU. However, scientific and technical support for organizing a surveillance program can be provided by the European Food Safety Authority (EFSA).
At the meeting it was concluded that in order to be able to perform surveillance of animal botulism cases, there has to be a reporting system established in each country, along with better and more efficient diagnostic tools; otherwise, the disease will continue to be underdiagnosed and underreported.

\section{Concluding Remarks}

How well prepared are we for future threats of botulism? Thanks to national and international research projects in the field, there are now fast and accurate diagnostic methods being established, and awareness is increasing in Europe. The AniBioThreat project is an excellent example of a project spanning country boundaries and thereby increasing communication and awareness in Europe. The aim is to continue the scientific collaboration between partners after the AniBioThreat project ends.

Different needs that have to be addressed to increase biopreparedness in Europe were defined at the workshop. Examples of such needs are a centralized commercial stock of botulinum antitoxins and vaccines, implementation of efficient toxin detection tools in European laboratories, and a surveillance system for animal botulism cases. It is also necessary to increase awareness among veterinarians working in the field with clinical cases and to provide guidelines for handling botulism outbreaks. Finally, there are indications that animal botulism is an emerging disease in Europe and should therefore be getting more attention from both a human and an animal health perspective.

\section{ACKNOWLEDGMENTS}

We would like to acknowledge all the participants at the workshop, especially the presenters, for their valuable contribution to this report. The workshop was supported by the Swedish Civil Contingencies Agency. This research was supported by and executed in the framework of the EU project AniBioThreat (Grant Agreement: Home/2009/ ISEC/AG/191) with financial support from the Prevention of and Fight against Crime Programme of the European Union, European Commission-Directorate General Home Affairs. This article reflects the views only of the authors, and the European Commission cannot be held responsible for any use that may be made of the information contained therein.

\section{REFERENCES}

1. Centers for Disease Control and Prevention. Possession, use, and transfer of select agents and toxins; biennial review. Final rule. Fed Regist 2012;77:61083-61115.

2. Hatheway CL. Toxigenic clostridia. Clin Microbiol Rev 1990;3:66-98.

Biosecurity and Bioterrorism: Biodefense Strategy, Practice, and Science 
3. Myllykoski J, Lindström M, Keto-Timonen R, et al. Type C bovine botulism outbreak due to carcass contaminated nonacidified silage. Epidemiol Infect 2009;137:284-293.

4. Jalava K, Selby K, Pihlajasaari A, et al. Two cases of foodborne botulism in Finland caused by conserved olives, October 2011. Euro Surveill 2011;16:20034.

5. Collins MD, East AK. Phylogeny and taxonomy of the foodborne pathogen Clostridium botulinum and its neurotoxins. I Appl Microbiol 1998;84:5-17.

6. AniBioThreat. Animal botulism in Europe: current status of an emerging disease. Abstracts of reports of botulism in animals over the last decade written by participants of the animal botulism workshop. Uppsala 2012. SVA Report No. 17.

7. Payne JH, Hogg RA, Otter A, Roest HI, Livesey CT. Emergence of suspected type D botulism in ruminants in England and Wales (2001 to 2009), associated with exposure to broiler litter. Vet Rec 2011;168:640.

8. Lindstrom M, Myllykoski J, Sivela S, Korkeala H. Clostridium botulinum in cattle and dairy products. Crit Rev Food Sci Nutr 2010;50:281-304

9. Advisory Committee on the Microbiological Safety of Food. Report on Botulism in Cattle. London 2005. http://www.food. gov.uk/multimedia/pdfs/botulismincattlereport1206.pdf. Accessed June 12, 2013.

10. Skarin H, Lindberg A, Blomqvist G, Aspan A, Baverud V. Molecular characterization and comparison of Clostridium botulinum type C avian strains. Avian Pathol 2010;39:511-518.

11. Hyun SH, Sakaguchi G. Implication of coprophagy in pathogenesis of chicken botulism. Nihon Juigaku Zasshi 1989;51:582-586.

12. Jeffrey JS, Galey FD, Meteyer CU, Kinde H, Rezvani M. Type $\mathrm{C}$ botulism in turkeys: determination of the median toxic dose. I Vet Diagn Invest 1994;6:93-95.

13. Hogg RA, Livesey CT, Payne JH. Diagnosis and implications of botulism. Farm Animal Pratice 2008;30:392-397.

14. Newton JR, Wylie CE, Proudman CJ, McGorum BC, Poxton IR. Equine grass sickness: are we any nearer to answers on cause and prevention after a century of research? Equine Vet I 2010;42:477-481.

15. Skarin H, Hafstrom T, Westerberg J, Segerman B. Clostridium botulinum group III: a group with dual identity shaped by plasmids, phages and mobile elements. $\underline{B M C}$ Genomics 2011;12:185.
16. Evans ER, Skipper PJ, Shone CC. An assay for botulinum toxin types $\mathrm{A}, \mathrm{B}$ and $\mathrm{F}$ that requires both functional binding and catalytic activities within the neurotoxin. $L$ Appl Microbiol 2009;107:1384-1391.

17. Boyer AE, Moura H, Woolfitt AR, et al. From the mouse to the mass spectrometer: Detection and differentiation of the endoproteinase activities of botulinum neurotoxins A-G by mass spectrometry. Anal Chem 2005;77:3916-3924.

18. Kalb SR, Moura H, Boyer AE, McWilliams LG, Pirkle JL, Barr JR. The use of Endopep-MS for the detection of botulinum toxins $\mathrm{A}, \mathrm{B}, \mathrm{E}$, and $\mathrm{F}$ in serum and stool samples. Anal Biochem 2006;351:84-92.

19. Hedeland M, Moura H, Baverud V, Woolfitt AR, Bondesson U, Barr JR. Confirmation of botulism in birds and cattle by the mouse bioassay and Endopep-MS. I Med Microbiol 2011;60:1299-1305.

20. Agren J, Sundstrom A, Hafstrom T, Segerman B. Gegenees: fragmented alignment of multiple genomes for determining phylogenomic distances and genetic signatures unique for specified target groups. PLoS One 2012;7:e39107.

21. Woudstra C, Skarin H, Anniballi F, et al. Neurotoxin gene profiling of Clostridium botulinum types $\mathrm{C}$ and $\mathrm{D}$ native to different countries within Europe. Appl Environ Microbiol 2012;78:3120-3127.

22. Directive 2003/99/EC of the European Parliament and of the Council of 17 November 2003 on the monitoring of zoonoses and zoonotic agents, amending Council Decision 90/424/EEC and repealing Council Directive 92/117/EEC.

Manuscript received December 20, 2012;

accepted for publication April 10, 2013.

Address correspondence to:

Hanna Skarin

National Veterinary Institute (SVA)

Department of Bacteriology

Ulls väg 2

Uppsala 75189

Sweden

E-mail: hanna.skarin@sva.se

\section{List of Presenters}

Andreas Rummel

Annica Tevell Åberg

Birgit Schwagerick

Bo Segerman

Caroline Le Marechal

Cédric Woudstra
Hannover Medical School, Germany

National Veterinary Institute, Sweden

Bovine Health Service, Germany

National Veterinary Institute, Sweden

French Agency for Food, Environmental and Occupational Health Safety, France

French Agency for Food, Environmental and Occupational Health Safety, France

(continued) 


\begin{tabular}{|c|c|}
\hline Dario De Medici & Istituto Superiore di Sanità, Italy \\
\hline Frank Gessler & Miprolab, Göttingen, Germany \\
\hline Gunilla Blomqvist & National Veterinary Institute, Sweden \\
\hline Hanna Skarin & National Veterinary Institute, Sweden \\
\hline Helge Böhnel & Miprolab, Göttingen, Germany \\
\hline Ian Poxton & University of Edinburgh, Great Britain \\
\hline Ibone Anza Gomez & Instituto de Investigación en Recursos Cinegéticos, Spain \\
\hline Jan Langeveld & Central Veterinary Institute of Wageningen, Holland \\
\hline Jeffrey Skiby & Technical University of Denmark, Denmark \\
\hline Joakim Ågren & National Veterinary Institute, Sweden \\
\hline Luca Bano & Istituto Zooprofilattico Sperimentale delle Venezie, Italy \\
\hline Miia Lindström & University of Helsinki, Finland \\
\hline Rickard Knutsson & National Veterinary Institute, Sweden \\
\hline Rozenn Souillard & French Agency for Food, Environmental and Occupational Health Safety, France \\
\hline Shunji Kozaki & Osaka Prefecture University, Japan \\
\hline Simon Hardy & Norwegian Veterinary Institute, Norway \\
\hline Viveca Båverud & National Veterinary Institute, Sweden \\
\hline
\end{tabular}

